\title{
MULTIPLE ORGAN DYSFUNCTION SYNDROME ASSOCIATED WITH MYCOPLASMA PNEUMONIAE INFECTION
}

\author{
Shu-Bo Zhai ${ }^{1}$, Dian-Bo Cao ${ }^{2}$, Hui-Xu ${ }^{1}$, Xin-Xiang Li ${ }^{1}$, Si-Rui Yang ${ }^{1 *}$ \\ ${ }^{1}$ Department of Pediatrics, First Hospital of Jilin University, Changchun, Jilin, P. R. China; ${ }^{2}$ Department of Radiology, First \\ Hospital of Jilin University, Changchun, Jilin, P. R. China.
}

Submitted: August 13, 2010; Approved: August 30, 2011.

\begin{abstract}
In this study, we report one case of a three-year-old boy infected with Mycoplasma pneumonia (MP) and presenting concomitant multiple organ damage of the heart, kidney, lung and liver, among others, together with a brief review for the diagnosis and treatment of MP infection with multiple organ dysfunction syndrome (MODS).
\end{abstract}

Key words: Mycoplasma pneumonia (MP); multiple organ dysfunction syndromes (MODS)

Among 16 mycoplasma species found in humans, Mycoplasma pneumonia (MP) is the only one that is recognized as a pathogen (19). MP is an important infectious agent of the upper and lower respiratory tracts and is endemic in most areas of the world, although more common in temperate zones. MP infections are usually most prevalent in closed environments such as the home, school and preschools. Mycoplasma pneumonia can spread through droplets and has a long latency period, remaining a potential source of infection. The infection rate of MP has varied from $2 \%$ (endemic) to $35 \%$ (epidemic) in Seattle, Washington in the United States (20), and also has been reported to range from $16.9 \%$ to $44.6 \%$ for young patients in several cities of China (8). MP infection occurs not only in children but also in young adults, the highest incidence occurring in children between 3 and 14 years old (4, 6). Diagnosis of MP infection is primarily by laboratory tests, including culture, antigen detection, polymerase chain reaction, complement fixation assay and serology. The IgM antibodies are usually detectable 7 to 10 days after infection (4).

MP infection is a sub-acute and gradual process, which usually lasts for a month or sometimes longer, excluding the incubation period (3). Although MP infection is usually selflimiting and constitutional symptoms like fever, headache and malaise resolve in about 10 days, antibiotic and supportive therapies are important (4). The clinical symptoms of MP infection usually start in the upper respiratory tract and then spread to the lower. Additional symptoms that are not respiratory tract-related may include diarrhea, liver damage, myocardial damage, anemia, allergic purpura, nephritis, encephalitis, among others $(2,18)$. However, it is rare for MP infection in infants to spread beyond the lung, or to concomitantly cause multiple organ dysfunction syndrome (MODS) with involvement of more than four organs. We report one case of MP infection associated with MODS,

*Corresponding Author. Mailing address: Department of Pediatrics, First Hospital of Jilin University, Changchun 130021, Jilin, P. R. China.; Tel.: 86-43188782560 Fax: 86-431-85654528.; E-mail: siruiyang@163.com 
together with persistent elevated MP-specific IgM antibodies in infant plasma.

A 3-year-old boy was hospitalized with symptoms of a 2day high fever. Before hospitalization, his body temperature was over $40{ }^{\circ} \mathrm{C}$, then decreased to $38{ }^{\circ} \mathrm{C}$ after oral administration of acetaminophen, but went up again. Occasional cough, but no shivering or rash was observed. On admission, physical examination revealed a body temperature $41.6{ }^{\circ} \mathrm{C}$ (Fig. 1A), pulse 150 beats per minute, respiration 32 breaths per minute, blood pressure $90 / 60 \mathrm{mmHg}$, poor general condition, shortness of breath, sallow complexion, weak pale conjunctiva, pharyngeal congestion, heart rate of 150 regular beats per minute. No positive signs of liver, spleen, or nervous system abnormalities were observed. Of note, he had rare cold occurrence and was otherwise normal.

The initial laboratory results showed that the white cell count was $11.2 \times 10^{9} / \mathrm{L}$ (normal: $4.0-10.0 \times 10^{9} / \mathrm{L}$ ) with $74.9 \%$ granulocytes (normal 50-70\%), and his red blood cell (RBC) count was $3.63 \times 10^{12} / \mathrm{L}$ (normal: 4.0-5.5 $\times 10^{12} / \mathrm{L}$ ). His hemoglobin level was $102 \mathrm{~g} / \mathrm{L}$ (normal: 110-160 g/L), and his platelets $\left(\mathrm{PLT}\right.$ ) were $150 \times 10^{9} / \mathrm{L}$ (normal: 100 to $300 \times 10^{9} / \mathrm{L}$; Fig. 1F). A urinalysis was remarkably positive for protein, but negative for blood cells. Creatinine (Fig. 1E) and blood urea nitrogen in plasma were $130 \mu \mathrm{mol} / \mathrm{L}$ (normal: $44-115 \mu \mathrm{mol} / \mathrm{L}$ ) and $27.9 \mathrm{mmol} / \mathrm{L}$ (normal: 3.2-7.0 $\mathrm{mmol} / \mathrm{L}$ ), respectively. Aspartate aminotransferase (AST) was 1015.3 U/L (normal: 8.0-40.0 U/L), and alanine aminotransferase (ALT) was 235.5 U/L (normal: 8.0-50.0 U/L; Fig. 1C). The creatine kinase (CK) was $205.8 \mathrm{U} / \mathrm{L}$ (normal: 25-200 U/L), and the isoenzyme CK2 (CK-MB) was 412.3 U/L (normal: 0-25.0 U/L; Fig. 1D). The agglutination test showed MP-specific IgM titers of 1:40 (normal: <1:40; Fig. 1B). The serum test for Epstein-Barr virus, cytomegalovirus, hepatitis B virus, hepatitis A virus, and hepatitis $\mathrm{C}$ virus were all negative. X-ray showed that the double pulmonary textures were enhanced and thickened. Color Doppler ultrasound showed an enlarged left ventricle (diastolic diameter $37 \mathrm{~mm}$ ) with systolic function decreased by
$42 \%$ (normal: $50 \%$ to $75 \%$ ) for ejection fraction (EF) and $19 \%$ (normal: $27 \%$ to $35 \%$ ) for fractional shortening (FS).

On admission, the patient was treated with the antiinfective agent cefuroxime, together with a combination of supportive therapies that included cardiac, kidney and liver protection, myocardial nutrition and microcirculation promotion. Over the course of 5 days treatment, his blood pressure reduced to $75 / 50 \mathrm{mmHg}$, and rash appeared on his neck, his RBC was $3.23 \times 10^{12} / \mathrm{L}$, HGB was $99 \mathrm{~g} / \mathrm{L}$ and PLT was $42 \times 10^{9} / \mathrm{L}$ (Fig. 1F). Liver functions had improved significantly with AST of 2562.6 U/L and ALT of 1058.3 U/L (Fig. 1C), while the level of protein in the urine remained remarkably abnormal. The MP-specific $\operatorname{IgM}$ antibodies in plasma increased to $1: 160$ (Fig. 1B).

The signs and symptoms described above partly indicated a MP infection and systemic inflammatory response syndrome (SIRS), which progressed to MODS. Based on the outcome of this first round of treatment, azithromycin and dexamethasone were administered, following by transfusion to expand blood volume, and dopamine and dobutamine to boost blood pressure. On day 8 , the patient's body temperature started to decrease and blood pressure to stabilize, the rash on his neck disappeared. In addition, the parameters of his blood, urine and biochemical assays improved (Fig. 1). Color Doppler ultrasound showed an enlarged left ventricle (diastolic diameter $34 \mathrm{~mm}$ ) and its systolic function was now $42 \%$ for EF and $24 \%$ for FS. On day 11, the results for his blood, urine and biochemical assays showed further improvement (Fig. 1) with a MP-IgM of 1:320 (Fig. 1B). On day 20, he was fully recovered (Fig. 1) and discharged with continuing therapy prescribed. At two weeks post-discharge his blood, urine and biochemical assay results were stable, and IgM was 1:160.

Early detection and diagnosis are critical for the treatment of MP infection in extra-pulmonary manifestations. Microbiological culture is the most reliable method for the diagnosis of MP infection. However, this technique requires highly restricted culture conditions and is a time-consuming 
procedure. More importantly, positive findings are very low due to few numbers of the pathogen found in patient samples. Thus, it is difficult using microbiological culture as a routine test for identification of the infection (17). Serological tests are still the gold standard to diagnose MP infection (9) although nucleic acid amplification tests (NAATs) on throat secretions are superior in sensitivity as compared to serology during the early phase of MP disease (12), or nested-PCR (N-PCR) techniques (21). The focus of these tests is the MP-specific serum antibody titers, which are produced after one week, peak at 2-3 weeks, and return to normal after 3-4 months. The disease is diagnosed by isolation of MP and significant rises in titer of complement-fixing antibody in paired sera (20). The antibody titers of acute and convalescent sera should be tested. MP infection can be diagnosed if MP-IgM titers are consistently high (1:160) or if, in the convalescent stage, they are up to 4 times that of the acute stage (10). In this case, the patient presented initially with a fever, then with a slightly positive MP-IgM, high numbers of blood leukocytes and neutrophils, and then subsequently continuing increased IgM levels confirming the MP infection (Fig 1B).

MP is a mycoplasma, in size between that of a virus and a bacterium. It is enclosed in only a membrane, with no cell wall. Some antibiotics, which only block the synthesis of a cell wall, such as $\beta$-lactams, therefore have no therapeutic efficacy in the treatment of MP infection. For this reason successive treatments of erythromycin or azithromycin, which interfere with protein synthesis, are the most effective (13). The therapeutic course of macrolide antibiotics in pediatrics is generally less than 2 to 3 weeks, and the treatment course lasts 3 to 4 weeks to prevent relapse due to premature withdrawal (11).

Until recently, infection and immunological injury were considered the main factors responsible for the development of MP infections. The adhesion protein in the cell membrane of MP enables adhesion to respiratory epithelial cells of the host, and prevents mucociliary clearance (18). At the same time, the mycoplasma releases metabolic products including ammonia, hydrogen peroxide and nerve toxins, among others. This results in the destruction of the mucosal epithelium (14). The pathogenesis of Mycoplasma pneumoniae infection is considered to be in part attributed to excessive immune responses. (16). In recent years, studies have shown that the antigen of the mycoplasma has a similar composition to that of the host cell membrane, which stimulates B cells to produce autoantibodies and leads to a pathological immune response. The antigen components also promote lymphocytes, monocytes, macrophages and other inflammatory cell aggregation, and releases cytokines and inflammatory mediators. At the same time, after activating the complement system, immune complexes produce neutrophil chemotactic factors, which attract a large number of white blood cells to reduce invasive lesions, and then release lysosomal enzymes causing destructive lesions (15).

As reported in the literature (18), the MP infection rate of organs other than lung occur in the range of $25 \%$ to $50 \%$, among which the most common involvement is the blood (50\%), followed by skin (25\%), gastrointestinal tract $(25 \%)$, bone, joint and muscle (14\%), the central nervous system (1\%$6.7 \%)$, and the cardiovascular system (1\%-8.5\%). In this case, we found lesions in the kidney in addition to those in the blood, cardiovascular system and liver. MP infection leads to concomitant pulmonary complications and is associated with SIRS, which, in turn, leads to MODS or even multiple organ failure (5). The process from SIRS to MODS is a continuous progression. SIRS can aggravate to MODS if untreated (1). In this case, a similar process occurred after MP infection, although there were no serious concomitant pulmonary complications. SIRS is an immune-related disease and a leading cause of morbidity and mortality. It is a clinical syndrome with signs and symptoms related to infectious and non-infectious events in which the immune system overresponds to inflammation. In theory, glucocorticosteroids may be indicated for MP pneumonia when there is rapid 
development to an acute stage, or lung disease and other complications. Lee et al. (7) reported a successful therapy for 15 cases of severe MP pneumonia patients for whom antibiotic treatment had failed, when prednisone was administrated.

In conclusion, we report a case with MODS caused by MP infection. The possibility of an associated infection with MP
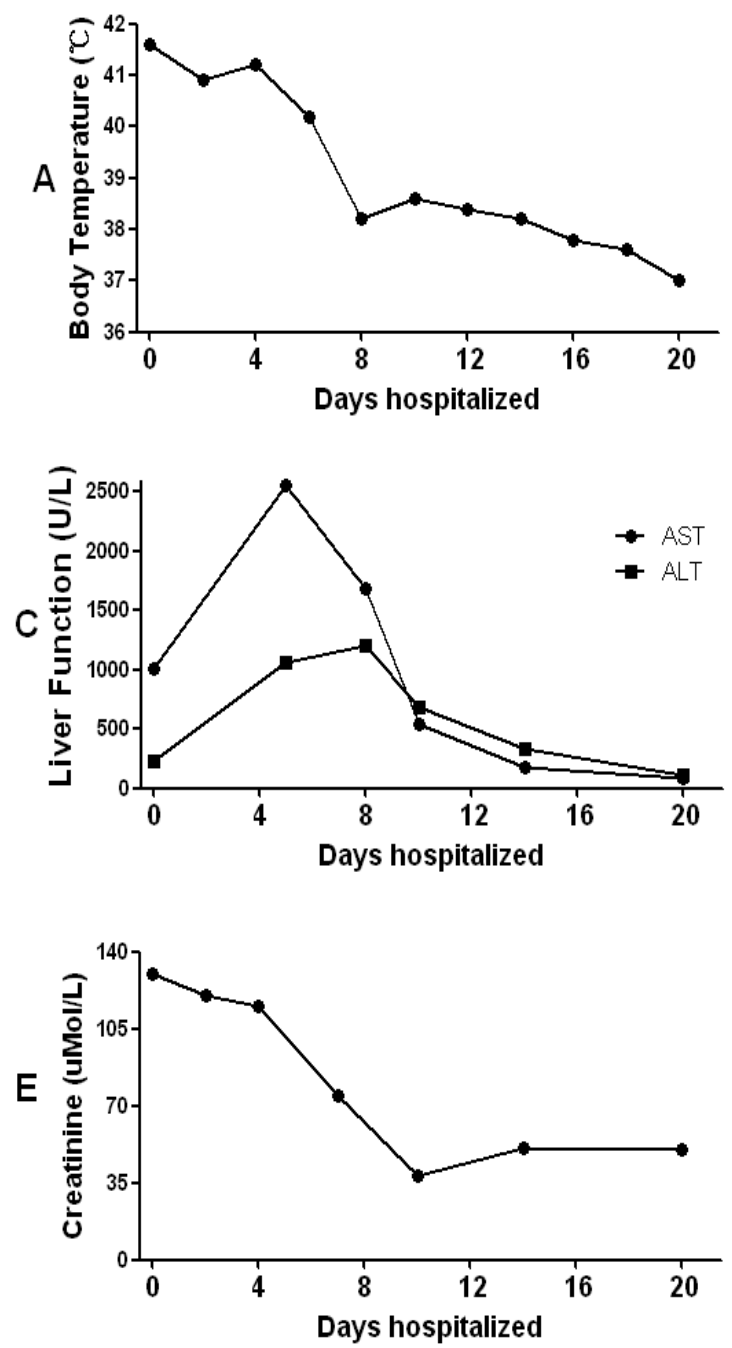

should be considered in children presenting with MODS. For the MP patient with rapid and serious infection at an acute stage, the administration of azithromycin antibiotics, together with additional glucocorticosteroid treatment and supportive procedures as necessary will result in a higher recovery rate.
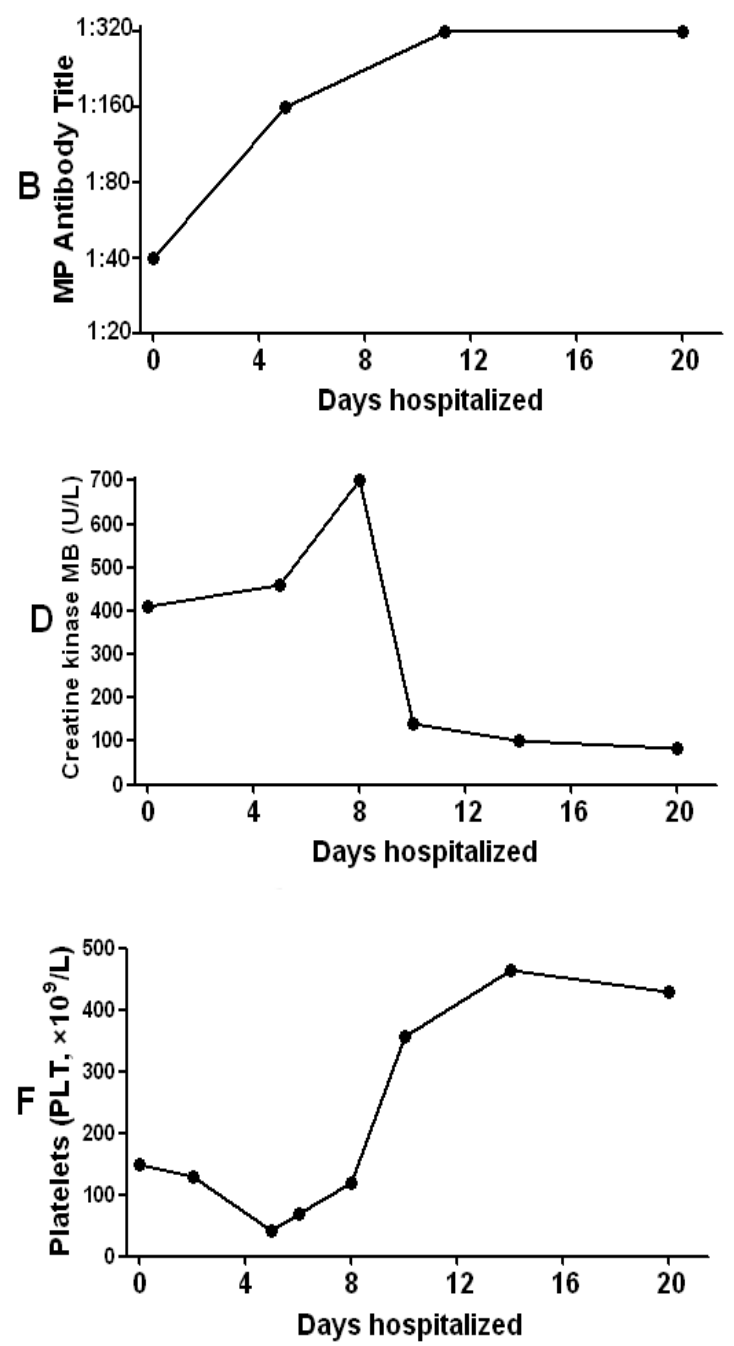

Figure 1. Measurement of body temperature, MP-IgM, ALT and AST, CK-MB, Cr activity and PLT in a patient infected with Mycoplasma pneumonia (MP).

The surveillance of body temperature (A), MP-IgM titre (B), ALT and AST U/L (C), CK-MB U/L (D), Cr $\mu$ mol/L (E) and PLT numbers/L (F) in a patient infected with Mycoplasma pneumonia (MP) in the indicated days after hospitalization. (MP-specific IgM titers detected by agglutination test, PLT by automated hematology analyzer (Sysmer XZ-2100, JAPAN), ALT, AST, CK-MB, and Cr by Automated Biochemistry Analyzer (BECKMAN SYNCHRON LX20, USA). The curves indicate the changes examined in the days indicated. 


\section{REFERENCES}

1. Bone, R.C. (1996). Immunologic dissonance: a continuing evolution in our understanding of the systemic inflammatory response syndromes (SIRS) and the multiple organ dysfunction syndromes (MODS). Ann. Intern. Med. 125 (8), 680-687.

2. Chang, A.; Neofytos, D.; Horn, D. (2008). Candidemia in the $21 \mathrm{st}$ century. Future Microbiol. 3 (4), 463-472.

3. Clyde, W.A., Jr. (1993). Clinical overview of typical Mycoplasma pneumoniae infections. Clin. Infect. Dis. 17 Suppl 1 S32-36.

4. Ferwerda, A.; Moll, H.A.; de Groot, R. (2001). Respiratory tract infections by Mycoplasma pneumoniae in children: a review of diagnostic and therapeutic measures. Eur. J. Pediatr. 160 (8), 483-491.

5. Han, X.H.; Liu, L.Y.; Jing, H.; Liu, T.Y.; Zhao, Y.Q.; Shang, Y.X. (2007). Changes of inflammation-associated factors in children with Mycoplasma pneomoniaepneumonia and concomitant systemic inflammatory response syndromes. Zhongguo Dang Dai Er Ke Za Zhi. 9 (4), 347-350.

6. Hassan, J.; Irwin, F.; Dooley, S.; Connell, J. (2008). Mycoplasma pneumoniae infection in a pediatric population: analysis of soluble immune markers as risk factors for asthma. Hum. Immunol. 69 (12), 851855.

7. Lee, K.Y.; Lee, H.S.; Hong, J.H.; Lee, M.H.; Lee, J.S.; Burgner, D.; Lee, B.C. (2006). Role of prednisolone treatment in severe Mycoplasma pneumoniae pneumonia in children. Pediatr. Pulmonol. 41 (3), 263-268.

8. Lu, D.; Liu, S.Q.; Zhuang, L.B.; Gong, S.Z.; Ruan, S.; Zhou, Y. (2006). Childhood upper respiratory infection caused by Mycoplasma pneumoniae. Zhongguo Dang Dai Er Ke Za Zhi. 8 (3), 205-207.

9. Martinez, M.A.; Ruiz, M.; Zunino, E.; Luchsinger, V.; Avendano, L.F. (2008). Detection of Mycoplasma pneumoniae in adult communityacquired pneumonia by PCR and serology. J. Med. Microbiol. 57 (Pt 12), 1491-1495.

10. Morozumi, M.; Hasegawa, K.; Chiba, N.; Iwata, S.; Kawamura, N.; Kuroki, H.; Tajima, T.; Ubukata, K. (2004). Application of PCR for Mycoplasma pneumoniae detection in children with community-acquired pneumonia. J. Infect. Chemother. 10 (5), 274-279.
11. Morozumi, M.; Iwata, S.; Hasegawa, K.; Chiba, N.; Takayanagi, R.; Matsubara, K.; Nakayama, E.; Sunakawa, K.; Ubukata, K. (2008). Increased macrolide resistance of Mycoplasma pneumoniae in pediatric patients with community-acquired pneumonia. Antimicrob Agents Chemother. 52 (1), 348-350.

12. Nilsson, A.C.; Bjorkman, P.; Persson, K. (2008). Polymerase chain reaction is superior to serology for the diagnosis of acute Mycoplasma pneumoniae infection and reveals a high rate of persistent infection. BMC Microbiol. 893.

13. Othman, N.; Isaacs, D.; Daley, A.J.; Kesson, A.M. (2008). Mycoplasma pneumoniae infection in a clinical setting. Pediatr. Int. 50 (5), 662-666.

14. Sekine, H.; Taguchi, H.; Watanabe, H.; Kawai, S.; Fujioka, Y.; Goto, H.; Kobayashi, H.; Kamiya, S. (2009). Immunological analysis and pathological examination of gnotobiotic mice monoassociated with Mycoplasma pneumoniae. J. Med. Microbiol. 58(Pt 6), 697-705.

15. Shenoy, V.D.; Upadhyaya, S.A.; Rao, S.P.; Shobha, K.L. (2005). Mycoplasma pneumoniae infection in children with acute respiratory infection. J. Trop. Pediatr. 51 (4), 232-235.

16. Shimizu, T.; Kida, Y.; Kuwano, K. (2007). Triacylated lipoproteins derived from Mycoplasma pneumoniae activate nuclear factor-kappaB through toll-like receptors 1 and 2. Immunology. 121 (4), 473-483.

17. Thurman KA, Walter ND, Schwartz SB, et al.Comparison of Laboratory Diagnostic Procedures for Detection of Mycoplasma pneumoniae in Community Outbreaks. Clin Infect Dis. 2009 May 1;48(9):1244-9.

18. Vervloet, L.A.; Marguet, C.; Camargos, P.A. (2007). Infection by Mycoplasma pneumoniae and its importance as an etiological agent in childhood community-acquired pneumonias. Braz. J. Infect. Dis. 11 (5), 507-514.

19. Waites KB. New concepts of Mycoplasma pneumoniae infections in children. Pediatr Pulmonol. 2003;36:267-278.

20. Waites, K.B.; Balish, M.F.; Atkinson, T.P. (2008). New insights into the pathogenesis and detection of Mycoplasma pneumoniae infections. Future Microbiol. 3 (6), 635-648.

21. Yamaguti, M.; Muller, E.; Piffer, A.; Kich, J.; Klein, C.; Kuchiishi, S. (2008). Detection of Mycoplasma hyopneumoniae by polymerase chain reaction in swine presenting respiratory problems. Braz. J. Microbiol. 39 (3), 471-476. 